\title{
Le retour du sexe dans le queer : Ici commence la nuit d'Alain Guiraudie
}

Enda MCCAFFREY, Université de Nottingham Trent

As soon as persons are posited, the war begins.

(Intimacies, 25)

\section{Introduction}

En 2014, Alain Guiraudie a publié son premier roman Ici commence la nuit (Prix Sade), dont l'action se déroule dans la région Languedoc en France. Il a été reconnu en tant qu'inspiration pour son précédent film L'inconnu du lac, primé à Cannes en 2013 (Prix Queer Palm). Gilles Heurtebise, le protagoniste et narrateur du roman de Guiraudie, arrive au terme de ses vacances d'été pendant lesquelles il a passé la majeure partie de son temps au lac local, véritable terrain de rencontre. Il a cependant créé une amitié insolite avec une famille venant des environs. Les événements prennent un tournant macabre au moment où Gilles vole, puis porte une paire de slips qui était étendue sur la corde à linge de la famille. Il éjacule dans le slip volé et le replace (humide) sur la corde à linge, guidé par la fausse croyance selon laquelle il n'aurait pas été vu. Cela mène à une enquête complexe et fantastique dirigée par un inspecteur de police sadique, à une romance entre Gilles et Pépé (l'homme de quatre-vingt-dix-huit ans, propriétaire du slip volé par Gilles), à un récit psychanalytique dans lequel la nature tordue des événements invite Gilles à questionner son train de vie d'homosexuel, plus particulièrement les mœurs légères qui l'ont guidé jusqu'ici, ainsi qu'un questionnement sur sa capacité à un jour s'engager dans une relation monogame.

Ici commence la nuit est une intervention opportune dans deux débats agitant aujourd'hui les théoriciens queer. Guiraudie base ses écrits sur une opposition à l'asexualité perçue dans le domaine de la théorie queer de nos jours, l'accusation d'abstraction, la désincarnation et l'institutionnalisation dont elle est souvent taxée. L'éthique inflexible de $N o$ Future de Lee Edelman croise d'autres réactions queer plus optimistes quant au futur, comme la valeur de transformation dans l'art de l'échec (Jack Halberstam) et l'idéalisme radical dans la théorie de la performance dans l'œuvre de José Esteban Muñoz. On pourrait suggérer que Guiraudie a lui aussi un intérêt pour le futur grâce au potentiel de la littérature en tant que fantasme, surtout dans la réinvention des dynamiques du désir queer. Je défends l'idée que l'originalité de Guiraudie (tout d'abord littéraire, puis cinématographique) réside dans sa façon de rendre le sexe queer dans l'espoir de traverser les modalités relationnelles dominantes des sexes, la monogamie ainsi que le narcissisme. À travers des procédés comme le cruising, le 
renoncement de soi (rédemption) et l'ascétisme, Guiraudie amène le désir homosexuel au-delà du sexe, la personne et le corps indiquant la durabilité du désir homosexuel porté sur le futur et la vie éternelle. Méthodologiquement, je souhaite situer le texte de Guiraudie dans une trajectoire queer spécifique reposant sur le travail de l'un des plus anciens architectes de la théorie queer, Leo Bersani, et son concept de relationalité impersonnelle. La relationalité impersonnelle est la dissolution et la dispersion du soi en faveur d'une « universal relatedness grounded in the absence of relations, in the felicitous erasure of people as persons »(Intimacies, 38). J'envisage également trois aspects de la jouissance lacanienne : la jouissance comme un manque (castration); la jouissance féminine (la jouissance en tant qu'Autre) liée à l'axiome lacanien « il n'y a pas de rapport sexuel »; et la jouissance comme lalangue, moteur lacanien de l'inconscient. Je montre comment l'homosexualité de Gilles éveille une polémique queer plus large selon laquelle une résistance aux constructions personnalisées du soi et de la jouissance expose la possibilité de l'existence relationnelle comme une alternative authentifiant le paradigme fondé dans ce que Bersani appelle «impersonal relationality ». De surcroît, j'illustre la manière dont Guiraudie reconnecte son lecteur avec les procédés bruts du désir queer, ses modes opératoires, ses motivations inconscientes, et l'optimisme renouvelé face à la négativité caractérisant la théorie queer de nos jours. Pour des raisons d'espace, et afin d'évaluer la profondeur et l'étendue théorique de la vision de Guiraudie, cette étude se limite à son roman. Cependant, les chevauchements entre le roman et le film sont significatifs et les spécialistes de la théorie et de la philosophie filmiques pourraient y trouver de l'intérêt.

\section{Relationalité impersonnelle}

La relationalité impersonnelle peut expliquer une partie de l'entrain libidineux qui définit Gilles Heurtebise. Cet entrain pourrait être fondé sur ce que Bersani appelle «the shattering of self »- la capacité qu'a la sexualité d'éclater les relations sexuelles, les transformant en une (non-)relationalité dépersonnalisée et désindividualisée :

I have been proposing that we think of the sexual - more specifically, of jouissance in sexuality - as a defeat of power, a giving up, on the part of an otherwise hyperbolically self-affirming and phallocentrically constituted ego, of its projects of mastery. Thus the subject enters into a Bataille-like communication with otherness, one in which the individuating boundaries that separate subjects [...] are erased (Is the Rectum a Grave ?, 109).

Le train de vie de Gilles, composé d'une multiplicité d'amants sans noms et de son implication dans des activités comme le cruising, est symptomatique de cet éclatement. L'éclatement est essentiellement associé avec la topologie des espaces ouverts (la foule, la ville, le bord du lac). 
Dans la première scène au lac, les noms ainsi que les visages des autres homosexuels sont effacés : «les inconnus » apparaissent derrière des arbres, se masturbant tout en « regardant » Gilles. La rue donne également la possibilité aux regards de se croiser (Guiraudie 108). Le cruising et son mode opératoire sont aussi une façon pour Guiraudie d'explorer l'impact de l'éclatement du soi et du sexe. Dans l'une des dernières scènes au lac, après le meurtre de Thibault Lombard, Gilles est interrogé par l'inspecteur de police. La scène pivote sur la subversion des hypothèses hétéronormatives quant au cruising. L'inspecteur doute, étant donné le temps qu'a passé Gilles à s'adonner au cruising, qu'il soit incapable de donner des noms, des numéros de téléphone ou d'autres détails sur les autres cruisers pouvant faire avancer l'enquête. Ce qui perturbe d'autant plus l'inspecteur, c'est que Gilles semble insensible à la mort d'un homme homosexuel local dans un endroit qui lui est si familier. Discréditant l'hypothèse d'une communauté homosexuelle très soudée, Guiraudie indique l'existence d'un autre type de fraternité homosexuelle fondée sur l'impersonnalité ainsi que l'aspect social du sexe :

Cruising, like sociality, can be a training in impersonal intimacy [...]. In cruising, we leave our selves behind [...]. Otherness, unlocatable within differences that can be known and enumerated, is made concrete in the eroticised touching of a body without attributes. A nonmasochistic jouissance $[\ldots]$ is the sign of nameless, identity-free contact - contact with an object I don't know and certainly don't love and which has, unknowingly, agreed to be momentarily the incarnated shock of otherness. In that moment we relate to that which transcends all relations ( $I S$ the Rectum a Grave ?, 61).

La relation de Gilles avec sa nouvelle famille renforce l'aspect « social » de l'impersonnel. Cela débute au moment où il admet se sentir à l'aise dans cette demeure, malgré le fait que les autres résidents se sont tout juste rencontrés. Même la torture terrifiante et sadique de Gilles par l'inspecteur de police dans la salle de bain ne soulève aucune récrimination ; en effet, la famille finit par se rassembler pour célébrer la libération de Gilles. Le fait de ne rien avoir en commun avec les membres de cette famille est la raison pour laquelle Gilles a de l'affinité envers eux. Guiraudie utilise l'assimilation de Gilles dans l'unité familiale pour subvertir les normes de la loyauté fraternelle, la rivalité ainsi que l'amitié, et explorer les formes relationnelles impersonnelles : «Je leur parlais de ma vie mais pas trop non plus et ils prenaient ce que je leur disais sans chercher à en savoir plus » (45). En l'absence d'un quelconque passé commun ou d'une curiosité mutuelle, Guiraudie crée une structure relationnelle alternative dans laquelle Gilles est le fils adoptif et contrecarre ainsi les normes œdipiennes en mettant en scène une liaison sexuelle entre lui et le grand-père (Pépé), tout en le faisant résister aux avances de la fille Cindy et de la mère Mariette. L'entrée de Gilles dans cette structure de 
substitution exotique éclate les relations sexuelles personnalisées et (hétéro-) normatives à tel point que l'aisance qu'il ressent en leur compagnie est mesurée par sa capacité à se masturber sans peur ou distraction : «Tellement je me sentais de mieux en mieux dans cette maison [...] j'ai commencé à me branler chez eux [...] Sans désir pour Pépé ou Mariette [...] Juste pour la chaleur de leur accueil, j'avais trouvé mon havre de paix »(45-46; je souligne). La masturbation sans objet est l'expression d'une confusion potentiellement bénéfique quant aux modes (sexuels) de connexion, une reconfiguration du relationnel qui peut nous aider à élaborer les modes d'être-au-monde par rapport auxquels le concept même d'identité pourrait être hors de propos. L'être relationnel pour Bersani n'est pas déterminé par le soi ou l'identité, mais par l'être inaccompli comme n'étant jamais plus que l'être potentiel. La nature idéaliste de ce contexte familial ouvre ce champ de potentialités.

Continuons notre analyse de la relationalité impersonnelle en adoptant une perspective psychanalytique à travers l'utilisation d'objets érotiques par Guiraudie, plus particulièrement les slips d'hommes et les pyjamas. Lacan affirmait dans les années 1970 qu' «il n'y a pas de rapport sexuel », signalant un tournant dans la psychanalyse des relations sexuelles humaines. En rejetant les fondations freudiennes du complexe d'Edipe, Lacan déclarait qu'il n'y a pas de formule sexuelle pour l'attraction entre différents êtres humains puisque la différence sexuelle n'est pas un concept. La jouissance pour Lacan n'est pas seulement sans limites, elle est aussi symptomatique d'un manque originel qui ne peut être comblé que dans un futur fantasmé et toujours reporté/différé. Cette théorie de la jouissance pourrait en partie expliquer la poursuite du plaisir sexuel entreprise par Gilles, incluant l'activité répétitive et frustrante qu'est le cruising. Or Lacan explore également les conséquences de cette poursuite du plaisir sexuel éternel, notamment les mécanismes de déplacement en l'absence d'un «rapport sexuel» (normatif). Lacan souligne deux éléments interconnectés importants à cet égard; la nature asymétrique de la relation entre l'Homme et la Femme signifie que la pulsion sexuelle n'est pas dirigée vers une personne entière, mais vers des objets tiers (un surplus de jouissance ou «plus-de-jouir » (17)). En d'autres termes, une relation sexuelle est établie entre un sujet et un objet (partiel - pour Gilles et Pépé, ce sont le slip et le pyjama). Lacan appelle cet objet l'objet $a$ et il a une double fonction : celle d'occuper la place d'une jouissance originelle manquante (mais aussi un partenaire/Autre, manquant) et celle de produire ce qu'il appelle un mathème de fantasme (SOa). L'Autre (Pépé) n'existe pas comme un sujet réel pour Gilles, mais comme un objet de fantasme et comme la cause de son désir.

La théorie de la jouissance de Lacan prolonge l'argument de la relationalité impersonnelle. Dans son auto-défense quant au vol du slip, Gilles admet que son besoin sexuel 
«est plus fort que [lui] »(Guiraudie 9). Cependant, peu de personnages le comprennent ou désirent l'accepter; Cindy essaye de le convertir à l'hétérosexualité en insistant qu'une fellation de sa part lui prouverait qu'il lui est possible de changer de préférence sexuelle. Le seul moyen qu'a Pépé pour intégrer l'idée que Gilles s'est masturbé dans son slip, est de connecter cet acte à lui-même directement. Aucune de ces interprétations ne considère la possibilité d'occlusion de l'objet du désir comme un sujet. La jouissance met les enjeux relationnels sur deux niveaux. Tout d'abord, cela renforce l'idée d'éclatement du sexe et la capacité de dépersonnalisation qu'a la sexualité selon Bersani, mais redistribue en même temps le désir sexuel autre part. Ensuite, en tant qu'incarnation de la relationalité impersonnelle, Gilles est confronté à des tentatives de rationalisation de son attitude. Ces tentatives sont majoritairement faites par l'inspecteur de police, qui requiert un spermogramme pour le slip sale dans le but d'identifier le coupable grâce à son ADN. Preuves et faits sont la devise de l'inspecteur, ils semblent diriger l'investigation policière également. Cependant, tandis que l'action continue (est suspendue), il est clair que Guiraudie utilise des rebondissements alambiqués pour exposer la direction obsessionnellement centrée sur l'identité de l'investigation, particulièrement dans son utilisation de certaines pratiques illicites. Nous découvrons que l'investigation n'a pas de limites sanctionnées puisque la discipline et la punition se déroulent en dehors de la loi dans une scène de torture policière graphique ; dans la salle de bain familiale, l'inspecteur attache Gilles à une chaise et enfonce le slip plein d'excréments de Pépé dans le rectum de Gilles par la force. Malgré la dimension extrême et graphique du sadomasochisme de la scène, l'inspecteur tente implicitement de rationaliser la jouissance de Gilles, puisqu'il le force non seulement à ingérer de nouveau sa jouissance en tant que punition, mais il force cette nouvelle ingestion en tant que catégorie de soi.

Le slip peut signifier plusieurs réactions à la relationalité impersonnelle. Gilles et l'inspecteur de police l'utilisent de manière différente. Gilles vole le slip de Pépé et se masturbe à l'intérieur, pas pour un quelconque désir d'une personne en particulier (pas même Pépé), mais à cause du frisson sensuel qu'il éprouve. Il insiste : « il n'y a rien de sexuel entre nous » (84). L'authenticité de Gilles contraste totalement avec l'approche obsessive de l'inspecteur dans ses tentatives d'attribuer à quelqu'un l'appartenance du slip, le menant à utiliser le slip comme instrument de punition, de torture psychologique et de séduction. En conséquence, l'inspecteur interprète l'anonymisation du slip par Gilles d'une mauvaise façon en la considérant comme l'expression d'un désir sexuel pour une personne en particulier. Cette opinion fausse la perspective de l'inspecteur envers son enquête et envers Gilles au point que, lorsqu'il se trouve impliqué sexuellement avec Gilles, l'inspecteur interprète faussement le slip 
de Gilles comme le signifiant de son désir (et de son amour) pour lui. Cette mauvaise interprétation se transforme en jouissance destructive de contrôle et de domination. Nous pouvons d'une part décoder les intentions de l'inspecteur en tant que manière d'interpréter le désir de Gilles en termes de catégorisation (la gérontophilie dans ce cas-ci). D’autre part, nous pouvons penser que l'inspecteur essaye de rationaliser le désir de Gilles : s'il n'est pas important que le slip dans lequel Gilles se masturbe appartienne à quelqu'un en particulier, pourquoi n'irait-il pas en acheter un au marché local pour se masturber ensuite ? Ces événements donnent la fausse impression d'une incompréhension sur la relationalité impersonnelle de Gilles. Loin d'être anonymes, les deux personnes auxquelles Gilles a dérobé des sous-vêtements dans le passé (Pépé et son ancien professeur, M. Escandolières) sont toutes deux connues de lui parce qu'il les avait déjà rencontrées et qu'il avait ressenti une attraction sensuelle instinctive envers elles. Cependant, ils ne sont pas des amis proches ou même des partenaires. Ils font partie de ce que Bersani appelle une sociabilité (sexuelle) plus large : «In cruising, I'm proposing another sexual model - once in which avoidance of relationships might be crucial in initiating, or at least clearing the ground for, a new relationality » (Is the Rectum a Grave ?, 59). L'idée cruciale est que Gilles n'est intéressé par une relation avec aucun d'eux ; il signale tout simplement sa jouissance d'une manière différente (en laissant du sperme dans leur sous-vêtement). À contrario, le fait qu'il porte le slip rouge de Gilles (couleur = identification du propriétaire), puis le dépose plus tard, trempé de sperme, sur le perron de l'appartement de Gilles, amène l'inspecteur à attribuer, d'une manière transparente et (mal)calculée, son désir pour un Autre spécifique (Gilles). Au lieu d'exciter l'intérêt de Gilles, l'action d'imitation malavisée de l'inspecteur (imitant le vol initial de Gilles du slip sur la corde à linge comme signe de rapprochement mutuel) inspire la névrose et la peur.

\section{Relationalité personnelle}

Guiraudie voit la jouissance comme une façon de connecter les gens de façon différente. À travers la jouissance et la sexualité, des personnes qui ne se connaissent pas se rapprochent. Allant à l'encontre de l'archétype d'une famille excentrique, Guiraudie établit la jouissance comme un registre authentique et durable de relationalité impersonnelle. Au contraire, l'inspecteur de police est omniprésent en tant qu'exécuteur de la relationalité personnelle à travers son invocation de Loi de l'État et de la Loi du désir. Son influence officielle est telle que tous les mouvements de Gilles dans la ville et hors de celle-ci ou dans la maison de Pépé sont sujets à sa surveillance et à son commandement ; les pérégrinations oniriques du roman signifient que, même en son absence, sa présence est envahissante. Cependant, lorsque 
l'inspecteur le séduit et finit par partager un appartement avec lui, Gilles se met à contester le train de vie dissolu qu'il a mené jusqu'ici. C'est une étape clé dans la manière progressive qu'a Guiraudie de cartographier l'homosexualité de Gilles, qui a débuté avec les visites qu'il rendait à Pépé dans son lit. Leurs rencontres (toutes dans ou sur le lit de Pépé) marquent le commencement d'un certain nombre d'échanges et d'interactions poussant Gilles à reconsidérer son histoire sexuelle et Guiraudie à affiner la dynamique entre relationalité impersonnelle et personnelle. Initialement, Gilles parvient à contrôler ses émotions et ses actions envers Pépé. Ce dernier a compris dès le vol de son slip que Gilles s'était masturbé à l'intérieur, et cela donne lieu à une série de questions pertinentes : «Tu te branlais dans mon slip, à deux pas de moi, mais tu pensais à moi ? » (55); «C'est moi qui te fais bander comme ça ? »(101; je souligne). Gilles confesse s'être masturbé, pas d'avoir pensé à Pépé : «J'aime être avec toi [...] Mais j'ai pas envie de toi » (55). Guiraudie nuance encore un peu plus : Gilles prend plaisir à «dormir avec » Pépé (plutôt distinct de « coucher avec»), mais se demande pourquoi il le fait, ne sait pas quoi faire après avoir dormi avec Pépé, supposant que «dormir avec » invoque une continuité relationnelle prenant la forme d'une conversation de chevet ou d'une cigarette après le coït. Gilles est « content dans l'intimité de Pépé » (63), mais s'abstient de l'embrasser malgré l'intensité de ses sentiments. Gilles dort dans le pyjama de Pépé, intoxiqué par l'odeur et la texture de sa fabrique, mais c'est une intimité séparée, via l'objet a. Cette deuxième pulsion libidinale (située dans le personnel, le gériatrique et l'idéalisme courtois) cause non seulement un important questionnement intérieur pour Gilles, mais représente également un défi pour la sociabilité et l'impersonnalité du désir sexuel de Gilles, qui a modelé sa vie ainsi jusqu'ici.

Pépé est surpris d'être la source du désir sexuel de quelqu'un d'aussi jeune. Il a internalisé cette attirance comme physique et sexuelle. Il attend d'être séduit par Gilles. Tout comme l'inspecteur de police, Pépé souhaite attribuer le vol de son slip à l'excitation d'un autre spécifique (Gilles). Tandis que les rencontres entre Gilles et Pépé croissent, ils se rapprochent, mais les chances d'un contact sexuel entre eux diminuent. Leur proximité affective a comblé la distance sexuelle entre eux. Malgré le fait qu'il désire le voir autant que possible, plus Gilles passe du temps loin de Pépé, plus ses sentiments envers lui s’intensifient et plus son désir de gratification sexuelle diminue. Ce qui est en jeu dans ce processus réciproque, c'est qu'il n'est pas question d'un quelconque manque d'excitation de Gilles envers le corps de Pépé ou de sa personne. C'est plutôt que, comme nous l'avons proposé, la jouissance de Gilles s'effectue par le biais d'un objet qui remplace le sujet. C'est également que dans le processus de remplacement objet/sujet lacanien, le vide entre l'objet et le sujet s'est tant creusé que la 
relation de Gilles avec Pépé devient liée à des préoccupations plus mentales que physiques. La jouissance a migré d'une fixation sur un slip (objet $a$ ) au sujet Pépé, puis à l'idée qu'il représente. Gilles a ainsi résisté jusqu'ici aux tentatives faites par Pépé et par l'inspecteur de police de personnaliser sa relationalité impersonnelle, mais la conséquence majeure est que sa résistance au contact sexuel a été déplacée dans l'esprit. Nous pouvons suggérer que Guiraudie utilise ce déplacement pour explorer une autre dimension de l'homosexualité de Gilles: «Penser à Pépé sereinement, sans image dans la tête, juste à l'idée de Pépé, il est là, dans mon cœur, il me manque, on se reverra bientôt. J'aime ça » (108; je souligne). L'émergence de l'idée de la personne possède une racine platonique dans la théorie de Platon à propos des formes (idées) dans lesquelles les formes non physiques représentent la réalité la plus exacte, contrairement à l'empirisme d'Aristote qui situe la chose/l'objet dans le là tout de suite. Nous pouvons lire ici le débat entre l'impersonnel et le personnel comme un dialogue entre la forme et l'empirisme. D'après Platon (cela est expliqué en détail dans La république et Phédon), les formes sont l'essence des objets/humains avec des associations non spatiales et intemporelles. Nous pouvons suggérer que Guiraudie invoque cette théorie de l'idée de Pépé dans le but de renforcer la thèse de la relationalité impersonnelle, pour qu'elle serve de tampon contre une personnalisation extrême. Cela annonce également la conclusion optimiste du roman, où l'amour dans sa signification platonique transcende le temps et la vie. De plus, l'idée de Pépé renvoie au code chevaleresque dans la façon dont l'amour courtois encadre la relationalité entre Gilles et Pépé plus tard dans le roman.

La relationalité personnelle dans le roman de Guiraudie n'est pas fondée sur l'être relationnel articulé par Bersani comme une sociabilité du sexe. Elle signifie plutôt un retour à l'idéalisation du personnel/de l'autre. Cependant, cela n'implique pas un retour au personnel personnalisé (comme dans l'attitude nécessiteuse personnifiée par l'inspecteur de police qui s'installe avec Gilles, ou effectivement dans celle de Pépé comme sujet lacanien du désir), mais un retour à la personne comme Idée. «L'idée de Pépé » alimente Gilles dans sa jouissance de l'autre et cette jouissance est répétée à travers une série de tropes déployés par Guiraudie. Le premier est la façon dont Guiraudie invoque et sabote la tradition occitane et hétéroïsée de l'amour courtois pour faciliter la re-homosexualisation de Gilles en amour - une décatégorisation de l'identité homosexuelle qui ensuite « libère l'amour de la demande de la personne » (Guiraudie 128). L'amour courtois était une discipline d'isolement. Par conséquent, l'amant (Pépé comme demoiselle) accepterait traditionnellement l'indépendance du prétendant (Gilles comme prétendant). Leur différence géospatiale respective soutiendrait cette liberté de mouvement négociée (l'un confiné au lit, l'autre constamment en déplacement). Le prétendant 
essaierait de devenir digne de son amant en agissant de manière honorable et en faisant tout ce qui lui est demandé par l'autre, se soumettant à une série d'actes et de tâches pour prouver son amour et son engagement. La satisfaction sexuelle laisserait souvent la priorité à l'attraction sexuelle comme point de convergence de la rencontre. Ici commence la nuit possède des aspects de la structure chevaleresque. Cependant, nous pouvons soutenir l'idée que Guiraudie détruit l'amour courtois en réimaginant ses codes et ses gestes. Cette destruction via des gestes fait appel à la théorie de la performance élaborée par Muñoz dans le contexte du théâtre, du travestissement et de la danse dans Cruising Utopia, mais aussi par Juana María Rodriguez dans son œuvre récente dans laquelle elle écrit : «Gesture functions as a socially legible and highly codified form of kinetic communication, and as cultural practice that is differentially manifested through particular forms of embodiment » (6). Guiraudie recourt à l'âge de Pépé, à sa vie d'amours non réciproques, à son registre courtois et, plus efficacement, à son utilisation de l'occitan dans ses échanges avec Gilles pour développer une autre façon de penser la relationalité et la jouissance. L'amour courtois devient une opportunité pour Gilles d'explorer l'esprit de Pépé : la façon dont il pense et communique, indirectement et avec des signes, les contours discrets et les gestes de son désir queer, ainsi que la manière dont cette interaction peut mener à une compréhension plus profonde de sa propre homosexualité en termes des pratiques enrichissantes d'amitié et de souci d'autrui (en dehors de la consommation sexuelle) qu'il démontre à la fin du roman. L'amour courtois se réfère également à l'idée platonique de compréhension mutuelle des connaissances entre deux collections de pensées et de positionnements (contrairement à deux personnes). Bersani contraste lui aussi la connaissance manifeste dans le sujet individuel (conduite par la jouissance) avec la connaissance comme un inconscient qui est réalisé non pas dans le désir, mais dans ce qu'il appelle « talk »: «This talk is the only imaginable form of a non-destructive jouissance, the jouissance of giving and receiving, through embodied language, the subjecthood of others » (Intimacies, 129).

La connaissance en tant que discussion inconsciente est exprimée en occitan. Cette langue est parlée à différents moments dans le roman et à plusieurs niveaux de maîtrise par divers personnages. Une officière de police salue Gilles en occitan, après avoir remarqué un roman (le roman célébré d'Enric Mouly E la barta Floriguet) sur la table de Gilles dans un café, dont il n'a pas achevé la lecture. Gilles parle un occitan «passable »; il le comprend mieux qu'il ne le parle. Pépé est celui qui a la meilleure connaissance de l'occitan, une habilité que Mariette et Cindy voient comme un signe de sa sénilité et de sa folie. D’une part, l'occitan est périphérique, un jaillissement de couleur régionale. D'autre part, il est central au jouis-sens du roman. L'occitan est utilisé pour la première fois au moment où Pépé répond à Gilles lors 
de leur première rencontre. Pépé emploie le mot « soscar » pour décrire son état d'esprit distrait. Le mot se traduit littéralement par «perdu dans les pensées », signe de la confusion de Pépé liée à son âge et d'une con(in)version plus profondément sexuelle et ontologique en progression. La fréquence de l'utilisation de l'occitan croît à mesure qu'ils se rencontrent, et l'évolution des échanges modifie notre analyse. Lorsque Pépé demande, en occitan, si Gilles pensait à lui lorsqu'il s'est masturbé dans son slip, celui-ci lui répond «Peut-être » en occitan, seulement pour confirmer quelques instants plus tard «Non» en français (104). Gilles ne parle pas couramment l'occitan, mais est clairement ému lorsque Pépé lui parle dans cette langue. En effet, Gilles est déçu quand Pépé reprend le français. La façon dont Pépé et Gilles communiquent leurs émotions est cruciale dans ces premiers échanges en occitan. Elle apparaît comme une langue secrète (connaissance inconsciente) ou comme ce que Lacan appelle lalangue (la jouissance inconsciente). Nous ne détectons pas seulement un plus haut degré d'honnêteté et d'authenticité lorsqu'ils communiquent en occitan ; le langage déployé par Pépé en particulier évoque un autre temps, lieu et état (d'esprit). L'occitan est sa langue maternelle, la « langue de son enfance » (136). Il désire Gilles ardemment en occitan : «je me languis de toi » (104). Le contact des mains (« il n'a pas envie de plus que ça [...] Juste la main [...] La main dans la main [...], j'en bande presque » (190)) n'est pas seulement un plaisir instinctif dans la compagnie de l'autre, mais aussi une maîtrise de soi noble et dépersonnalisée qui fuit l'indulgence requise d'un dénouement sexuel.

Cette courtoisie est également incarnée par l'un des anciens amants de Gilles. Paul voulait vivre avec Gilles, mais lorsque ce dernier a refusé, il s'est suicidé. Des réflexions sur Paul mènent au troisième rêve d'une série, dans laquelle Gilles rencontre Paul près d'une rivière. Pendant la conversation en occitan, Paul critique Gilles pour sa promiscuité. Il insiste également sur son désir d'être avec un autre homme (pas un fantôme) «en chair et en os » (234). Gilles répond en disant qu'un fantôme peut devenir « de chair et d'os ». Cet échange onirique est construit de nombreuses manières sur une trajectoire plus large du roman. Il place l'impersonnalité relationnelle de Gilles en contraste avec la fidélité monogame de Paul. La référence aux fantômes et le présage de chair/d'os annonce la transformation solitaire de Gilles à travers la renonciation de soi, et encadre l'externalisation de la jouissance en tant que lalangue. Le fait que cette transformation est également exprimée dans la langue « morte ${ }^{1}$ » qu'est l'occitan, donne vie à l'importance de l'occitan comme la véritable expression de l'inconscient (lalangue), ainsi qu'aux traditions et aux connaissances liées à l'amour courtois. L’importance de parler une langue «morte » est soulignée lorsque, après la crémation de Paul, Gilles retourne à son appartement et y trouve l'inspecteur de police en train de lire le roman 
d'Enric Mouly. C'est un autre moment clé pour comprendre le renoncement de soi effectué par Gilles. En réponse aux questions de l'inspecteur qui lui demande pourquoi quelqu'un désirerait continuer à parler une langue «morte » comme l'occitan, Gilles se lance dans une défense culturelle du passé. L'occitan n'est pas seulement la langue de ses parents, mais c'est aussi un moyen de maintenir la survie d'un «peuple ». Il insiste sur le fait que l'étude et la lecture de l'occitan sont une manière de partager une culture et de se connecter à une façon de vivre et de penser. Le sous-entendu est mis en lumière lorsque l'inspecteur demande si c'est cette manière de penser qui a provoqué son attirance envers Pépé, ce à quoi Gilles répond : « Oui, Pépé me touche plus en Occitan » (259). La réponse aliène l'inspecteur pour un certain nombre de raisons, que nous allons à présent considérer. Elle le sépare de Gilles (un amant potentiel) ; elle est une façon de considérer l'inspecteur comme un homme sans culture et jaloux ; elle renforce le lien linguistique, culturel et subliminal entre Gilles et Pépé et elle souligne considérablement l'idée d'impuissance chez l'inspecteur, qui peut peut-être imposer sa volonté sur Gilles, mais qui ne peut empêcher une langue «morte (l'amour) d'être parlée, aussi inadéquatement qu'elle le soit. De la même manière, la connaissance de l'occitan, une métaphore pour lalangue inconsciente de la jouissance, transperce le système visiblement cohérent de la langue française - textuellement, juridiquement, institutionnellement - dans le but de souligner les incohérences et les mauvaises utilisations qui discréditent le locuteur et ses idées dans le roman.

Les politiques culturelles et sexuelles de l'utilisation d'une langue «morte » attirent également notre attention sur un certain nombre de problèmes, qui gonflent le bagage homosexuel de Gilles et le positionnent comme une figure de positivité queer. La relation de désir entre Gilles et Pépé n'est jamais concrétisée en termes d' «homosexualité ». Leur désir réciproque va sans dire. Vers la fin du roman, Gilles est mis sous pression par d'autres individus pour des raisons d'honnêteté et de transparence pour que son homosexualité soit révélée (verbalisée) à Pépé, qui, de son côté, reste déphasé par la révélation. Le seul commentaire de ce dernier est de demander pourquoi il lui dit cela maintenant - signifiant que leur désir l'un pour l'autre a déjà été scellé dans lalangue de la jouissance et les idiolectes de l'amour courtois ; leur désir n'a ainsi pas besoin d'être verbalisé (et surtout pas en occitan). De façon significative, Gilles fait son coming out en français ; le faire en occitan serait d'après lui « ridicule » (189). La distinction est saillante. La divulgation publique de l'homosexualité est banale de nos jours. Le mot gay fait partie du lexique mondial, détournant des catégorisations identitaires et des statuts de personnes que la théorie de l'être relationnel de Bersani réprimanderait. D'une part, en confessant son homosexualité en français, Gilles se soumet partiellement à cette orthodoxie identitaire, bien qu'il admette avoir été mis sous pression pour 
faire cela. D'autre part, en disant qu'il serait ridicule d'utiliser l'occitan pour confirmer son homosexualité, il défend l'occitan en le considérant comme lalangue libre de la jouissance sans discrimination, libérée du conscient et des formations discursives du genre et de l'identité. L'occitan, en tant que lalangue, nous renvoie à la notion de Bersani « homoness » (Homos, 13) et à la théorie des formes de Platon, toutes deux étant la pratique de la communication, non pas entre des personnes, mais comme Bersani l'écrit : « of forms, as a kind of universal solidarity not of identities but of positionings and configurations in space, a solidarity that ignores even the apparently most intractable identity-difference»(Is the Rectum a Grave ?, 44). La langue «morte » qu'est l'occitan recadre la relationalité non intime, réhabilitant le personnel dépersonnalisé dans un contexte plus large d'amour et d'éternité courtois : «une idylle amoureuse sans sexe, un désir qui se concrétiserait ailleurs que dans le cul, qui ne se concrétiserait d'ailleurs pas, et qui du coup s'éteindrait jamais. Un désir éternel » (Guiraudie 140). En contraste avec la jouissance destructive de l'inspecteur de police, le train de vie anciennement libertin de Gilles avec ses promesses de gratifications sexuelles et le récit de négativité queer dominant, le roman de Guiraudie semble suggérer des manières alternatives pour entretenir le désir homosexuel (l'amour) grâce à une relationalité post-désir dans laquelle le désir d'appropriation du désir de l'autre n'existe plus. Bersani considère cela comme «the new ascetic pleasure of all-inclusive impersonal relationality 》(Intimacies, 29).

La défense de Gilles (et de Guiraudie) de l'occitan reflète également une inquiétude quant à la transmission et la préservation de la culture et de l'art, une perspective historique sur le rôle de l'héritage culturel, de la connaissance intergénérationnelle, le devoir d'être concerné par le passé et une résistance courageuse contre les tentatives faites pour le discréditer. Cette résistance consolide l'idée d'une culture qui serait préservée et entretenue à travers des actes discursifs (pas des institutions) qui à leur tour renforcent l'importance qui doit être attachée à la relation queer entre Gilles et Pépé. Pour Guiraudie, il est d'une importance culturelle et politique que la tradition de l'amour courtois et ses valeurs d'honnêteté, de vérité et de respect soient non seulement préservées mais aussi réinventées, incarnées et retransmises par un récit incluant des «homosexuels » en tant qu'acteurs principaux. Dans le même esprit, parler et homosexualiser en même temps une langue «morte» permet à Guiraudie d'explorer un discours alternatif à la jouissance corrosive de la théorie queer de No Future. À l'encontre d'un arrière-plan de gendarmerie corrompue et vindicative envers ceux qui cherchent à préserver de telles traditions et jalouse de ceux qui l'incarnent, Gilles émerge comme une figure de « redemption » (dans les termes de Bersani), nouveau Lazare ; Pépé et l'occitan ouvrent la voie à une homosexualité compassionnelle, chance pour Gilles d'obtenir sa propre rédemption en 
dehors de l'idée d'hyperbole de soi. Dans la tradition de courtoisie, c'est une opportunité de mettre l'aspect sexuel de côté, de prendre du plaisir et d'effectuer des tâches qui donneraient une certaine estime, à lui et à Pépé.

Le roman de Guiraudie défie l'éthique de négativité queer d'Edelman dans la mesure où il contient l'idée d'un futur. Pour Edelman, le futur se définit en termes de « reproductive futurism », privilégiant l'hétéro-normativité (2). Le queerness d'Edelman (qui protège le sinthomosexuel de l'ordre Symbolique du futurisme) est cependant restreint par sa «structural position » définie par la pulsion de mort. Puisqu'il n'existe pas de futur au-delà de la jouissance pure attachée à cet acte de rejet, le queerness d'Edelman invalide la valeur d'usage, le jugement moral et l'engagement émotionnel. Le queerness dans l'œuvre de Guiraudie n'est pas enfermé dans une structure ou une identité, mais devient une sexualité - un acte d'être ou de devenir dans la terminologie de Bersani - avec la capacité de radicaliser le Symbolique et de s'autotransformer. La scène finale du lit de mort, conduite dans un occitan fluide, permet de résumer ce potentiel transformatif à travers des trajectoires impersonnelles et personnelles antagonistes dans l'histoire homosexuelle de Gilles. La scène fusionne les forces indéniables qui ont généré cette relationalité impersonnelle avec cette nouvelle dévotion et cet engagement inspirés par Pépé. Le narcissisme impersonnel (contrepoint de Bersani à la relationalité impersonnelle) revient brièvement et graphiquement avec l'intention de l'inspecteur de police de violer et de castrer Gilles, rallumant ainsi en Gilles des sentiments de promiscuité sexuelle : «je peux pas dormir dans les bras d'un seul homme, j'ai toujours besoin d'un troisième et je me demande quand est-ce que tout ça finira, que je puisse enfin dormir tranquille» (283). Toutefois, ce retour est éclipsé par l'autotransformation de Gilles. Tandis que Pépé meurt dans le lit à côté de lui, Gilles s'occupe de lui et nettoie les excréments de son rectum. Il lui donne également du plaisir avec ses doigts, mais ne va pas jusqu'à la pénétration anale. La pénétration et l'éjaculation (moment climatique de la sexualité qui était autrefois tout ce pour quoi il vivait) sont remplacées par une suspension négociée d'une extrémité sexuelle à sa jouissance. Par l'image contenue dans « reste à bander» (avec le sexe en érection contre les fesses de Pépé en permanence - similaire en importance épistémique à l'utilisation par Bersani de la « figure de proue » de Genet dans Homos) - Guiraudie étend la chronologie homosexuelle de Gilles. Dans cette image, Gilles découvre une intensité sexuelle différente au-delà du sexe (une «grande vérité ») en compagnie de Pépé, nu, et dans une caresse mutuelle.

En suspendant la pénétration anale et la jouissance de Gilles en tant qu'orgasme, Guiraudie transfère le désir sexuel au-delà de sa demande d'immédiateté et de personnalisation, le transforme en un désir qui est entretenu après le moment, après la mort et pour l'éternité. 
Cette survie du désir queer comme un éternel au-delà du soi, de la castration et de la mort est une contestation évidente de la finitude fatale qui caractérise l'éthique queer de la mort défendue par Edelman, pour qui le « sinthomosexual is predetermined by a radical selflessness and unliveable desire that has its discourse of intelligibility as its own promising fatality [...], the ethical task for which queers have been singled out »(101). Le roman de Guiraudie défie cette éthique queer. Le renoncement à la jouissance, représenté par la castration de Gilles, est l'apothéose de son autotransformation. Culmination de l'éclatement du sexe, cette relationalité impersonnelle est éternisée à travers sa castration - l'acte suprême symbolique, d'après Bersani, de congédiement de soi avec le sexe en faveur du sexuel comme "a model of ascesis" (Intimacies, 97), dont nous pouvons remarquer certains aspects dans l'autosacrifice et le renoncement de soi de Gilles pour Pépé. Guiraudie élève Gilles au-delà de la catégorie du soi ; celui-ci prend une position morale différente, devient « a universal loved object of sacrifice » (96) et un héros dans le sens réel du roman mettant en scène l'amour courtois. Alain Badiou fait écho au modèle du désir ascétique de Bersani dans sa théorie de l'amour inspirée de Lacan. L'amour, pour Badiou, remplit l'absence de «pas de rapport sexuel» de Lacan. Tandis que la jouissance demeure asymétrique chez Lacan, la théorie de l'amour de Badiou privilégie la différence d'autrui (l'impersonnel) plutôt que l'identité de l'un (le personnel). En cela, Bersani et Badiou défient les préconceptions de fidélité (à UNE personne), exaltant plutôt l'universel et l'éternel : «L'amour [...] s'adresse à l'être même de l'autre, à l'être tel qu'il a surgi, tout armé de son être » (Badiou 25). La vie de Pépé a changé l'homosexualité de Gilles. La mort de Pépé l'a transfiguré : «Je laisse ma tête aller contre la poitrine de Pépé. J'ai encore envie de lui [...] Ça y est, je le tiens mon désir éternel » (285).

\section{Conclusion}

Ici commence la nuit appartient à une tradition de pensée queer sur la (non-)relationalité qui remonte à Hocquenghem et à Foucault et qui continue à façonner le «faire-le-monde queer » d'Edelman, d'Halberstam et de Muñoz. La compréhension de la (non-)relationalité comme un choix éthique est intrinsèque à cette tradition. Invoquant une importante position théorique et ontologique enrichie par le travail de Leo Bersani, le roman de Guiraudie (et son adaptation cinématographique) nous rappelle le lien entre sexualité et être. La promiscuité, le fétichisme et la jouissance ne sont pas des manifestations passives d'un inconscient inexplicable ; elles ne sont pas non plus définies par leur différence par rapport à un ordre ontologique hétéronormatif. Au contraire, l'homosexualité de Gilles Heurtebise dévoile une ontologie de l'être cohérente fondée sur le reniement des catégories de sexe et de soi. Ce 
reniement implique de nombreux aspects, allant de la relationalité impersonnelle et dépersonnalisée jusqu'aux détours créés par l'objet $a$. D'un œil critique, le reniement n'est pas une simple digression stylistique mais, comme Bersani l'affirme, il possède une «swerve» éthique persistante que nous pouvons cartographier à travers le texte de Guiraudie puisque ce dernier détourne notre attention des normes œdipiennes, reproductrices et sexuelles. C'est une embardée qui trouve sa rédemption dans la jouissance ascétique de la castration et la désinvestiture de soi. La contribution de Guiraudie aux écrits et aux pensées queer est distincte à cause de ces aspects. D'une part, le récit fantasque (incorporant une intrigue fabuleuse, un désir subliminal et une courtoisie queer) permet à Guiraudie de s'éloigner du réalisme et d'explorer un éternel queer plus large en tant que nouveau mode de radicalisme esthétique qui peut libérer les politiques et théories queer de leurs actuelles emprises dystopiques. D'autre part, tandis que l'éternel queer peut sembler mener négativement à un futur dans la finitude de la castration et la mort, c'est précisément dans cette finitude que Guiraudie extrait le pouvoir transformatif et (non-)relationnel de l'ascèse. À travers la castration, l'amour de Gilles est immortalisé : «It is as if love were pure once the subject absents himself from it, once this love without a subject is settled on its object and is itself absorbed into its object » (Intimacies, 53).

\section{Bibliographie}

Badiou, Alain. Éloge de l'amour. Paris : Flammarion, 2009.

Bersani, Leo. Is the Rectum a Grave? And Other Essays. Chicago : The U of Chicago P, 2009. ---. Homos. Cambridge, MA : Harvard UP, 1996.

Bersani, Leo et Philip Adams. Intimacies. Chicago : The U of Chicago P, 2010.

Edelman, Lee. No Future : Queer Theory and the Death Drive. Durham : Duke UP, 2004.

Guiraudie, Alain. Ici commence la nuit. Paris : P.O.L., 2014.

---. L'inconnu du lac. DVD, 2013.

Halberstam, Jack. The Queer Art of Failure. Durham : Duke UP, 2011.

Muñoz, José Esteban. Cruising Utopia. The Then and There of Queer Futirity. New York : New York UP, 2009.

Lacan, Jacques. Le séminaire. Livre XX. Encore, 1972-73. Paris : Seuil, 1975.

Rodriguez, Juana Maria. Sexual Futures, Queer Gestures. New York : New York UP, 2014.

\section{Note}

${ }^{1}$ En fait, l'occitan n'est pas (encore) une langue « morte », mais une langue minoritaire en danger. (NdIR) 